\title{
Functional characterization of human variants of NFKBIA: a key regulator of immune responsiveness implicated in susceptibility to infectious and inflammatory disease
}

\author{
Salman Ali, Aaron Hirschfeld, Rachel Victor, Edgardo S Fortuno III, Tobias R Kollmann, Stuart E Turvey \\ From AllerGen NCE Inc.'s Fifth Annual Research Conference: Innovation from Cell to Society \\ Québec City, QC, Canada. 7-9 February 2010
}

\section{Objective/purpose}

Genetic association studies have identified several polymorphisms in genes of the innate immunity cascade that appear to influence susceptibility to asthma and other inflammatory diseases. However, most candidate genes have not been functionally characterized for their impact on human immune responsiveness. An excellent candidate for functional investigation is NFKBIA which encodes $\mathrm{I} \kappa \mathrm{B} \alpha$-the major negative regulator of $\mathrm{NF} \kappa \mathrm{B}$. Single nucleotide polymorphisms (SNPs) in the promoter region of NFKBIA have been implicated in various infectious and inflammatory diseases. Specifically, the linked promoter SNPs rs2233406, rs3138053 and rs2233409 have been implicated in sarcoidosis, trachoma, acute respiratory distress syndrome, invasive pneumococcal disease, Graves' disease and respiratory syncytial virus. We investigated the mechanistic and functional impact of the promoter variants of NFKBIA on human immune responsiveness.

\section{Methods}

Using a coding SNP that was in high linkage with NFKBIA SNPs rs3138053/rs2233406/rs2233409, we designed and validated an allele-specific PCR assay that could detect subtle differences in allele ratios between the major (ACC) and minor (GTT) promoter variants of SNPs rs3138053/rs2233406/rs2233409. Peripheral blood mononuclear cells of homozygous (ACC/ACC) and heterozygous (ACC/GTT) individuals were stimulated with LPS and live cultures of Streptococcus pneumoniae (serotype 14) for 3 and 4 hours. PBMCs of NFKBIA homozygotes and heterozygotes were stimulated with various Toll-like-receptor (TLR) ligands of the innate immune cascade to assay for differences in the innate immune response.

\section{Findings}

NFKBIA heterozygotes (ACC/GTT) displayed 1.21 (1.14$1.2795 \%$ CI $)$ - 1.26 (1.18-1.34 95\% CI) fold higher expression of the major allele transcript (ACC) relative to the minor allele transcript (GTT). At 3 hours post stimulation, NFKBIA homozygotes (ACC/ACC) produced higher level of NFKBIA mRNA than heterozygotes (ACC/GTT) following stimulation with LPS (1.4 fold, $\mathrm{p}=0.0095$ ) or $S$. pneumoniae (1.51 fold, $\mathrm{p}=$ $0.024)$. Higher TNF- $\alpha$ secretion was seen from the peripheral blood mononuclear cells (PBMCs) of heterozygotes (ACC/GTT) as compared to homozygotes (ACC/ ACC) when stimulated with Pam3CSK4 (2.29-fold increase; $\mathrm{p}<0.01)$ and 3M-002 (3.30-fold increase; $\mathrm{p}<$ $0.001)$.

\section{Deliverables}

We have shown that the observed association of NFKBIA variants with infectious and inflammatory conditions has functional consequences. Individuals heterozygous for SNPs rs3138053/rs2233406/rs1050851 display allelic imbalance, reduced levels of NFKBIA expression, as well as a hyper inflammatory innate immune response. 


\section{Relevance}

Functional genomic studies such as this will help realize AllerGen's goal of 'discovery of the causes of, and ways to prevent, control or eliminate allergic and related immune diseases' by:

- Generating convincing evidence that the genetic variant is functionally relevant and likely to contribute to the development of the clinical phenotype.

- Providing insight into the mechanism underlying the genetic association and, therefore, greatly enhancing our knowledge of the disease pathogenesis.

- Identifying molecular pathways that can be targeted to prevent or treat allergic disease. 